DOI https://doi.org/10.30525/978-9934-26-113-8-37

\title{
THE LEVEL OF C3 AND C4 COMPLEMENT COMPONENTS IN RATS AFTER THE ADMINISTRATION OF MYASTHENOGENIC SERA WITH DIFFERENT CYTOTOXICITY
}

\author{
Lavinska O. V. \\ Ph.D. in Biology
}

Senior Researcher of diagnostic laboratory with immunoenzym and immunofluorescence analysis

State Institution Zaycev V. T. Institute of general and urgent surgery of National academy of Medical sciences of Ukraine;

Associate professor at the Department of Molecular Biology and Biotechnology

V. N. Karazin Kharkiv National University

Klimova O. M.

D.Sc. in Biology, Professor

Head of diagnostic laboratory with immunoenzym and immunofluorescence analysis

State Institution Zaycev V. T. Institute of general and urgent surgery of National academy of Medical sciences of Ukraine;

Professor at the Department of Molecular Biology and Biotechnology

V. N. Karazin Kharkiv National University

Kharkiv, Ukraine

Introduction. Antigens in the body are susceptible to sensitization and immune restructuring. This is manifested by the accumulation or specific oppression and inhibition of the immune response. The influence of various cytotoxic factors contained in the serum leads to changes in key events in the development of immunogenicity - antigen processing and presentation, which are carried out by phagocytic antigen-presenting cells (neutrophils, dendritic cells, macrophages and B cells). One of the main participants in the processing and presentation of the antigen are opsonizing factors of the complement system [1, p. 2-3].

Processes of antigenic perception in response to foreign antigens are provided by different receptors, such as Fc-receptors of immunoglobulins, complement proteins receptors (CR), toll-like receptors (TLR), 
immunoglobulin's super families, heat shock proteins receptors and others [2, p. 611].

The ability of different specificity antigens to elicit an immune response is determined by the antigens immunogenicity degree, which depends on the antigen molecular characteristics, the antigen kinetics in the body and the macroorganism reactivity. The inflammatory process is characterized by the immune cells activation, changes in vascular permeability and the proinflammatory mediators' synthesis, including cytokines, chemokines, lipid mediators, steroids, growth factors and the complement system protein synthesis [3, p. 7205]. In patients with myasthenia gravis, a severe neuromascular disorder, stress triggers lead to disturbances at all levels of regulation. This contributes to the formation of myasthenogenic factors, the heterogeneous nature of which remains unknown. Therefore, the influence degree of serum myasthenogenic factors from patients with various forms of myasthenia on the complement system activation as indicator of innate immunity in animals after metabolism is of interest.

The aim of the work is to study the changes in the $\mathrm{C} 3$ and $\mathrm{C} 4$ complement components content in rats after immunization of myasthenogenic sera with cytotoxicity varying degrees.

Materials and methods. The experiments were carried out on 3-monthold male Wistar rats (weight 170-190 g). The animals were kept in the standard conditions of the vivarium; food and water were got ad libitum. The studies were carried out in accordance with the provisions of the European Convention for the Protection of Vertebrate Animals Used for Experimental and Other Scientific Purposes (Strasbourg, 1986).

Serums of patients with thymus-independent myasthenia ( $\mathrm{M}$ - myasthenia without thymus changes) and thymus-dependent myasthenia (MH - myasthenia with thymus hyperplasia and MT - myasthenia with thymoma) were used for immunization. The cytotoxicity degree of selected sera was previously determined in the analysis using unicellular algae - the bioindicator Dunaliella viridis by the cytotoxicity coefficient $\mathrm{Cc}$ (the test system for determining integrated cytotoxicity was developed at the Research Institute of Biology, V.N. Karazin, Kharkiv National University). It was evaluated: $\mathrm{Cc}=$ 5.0 - low degree of cytotoxicity (M), Cc $=7.0$ - average degree of cytotoxicity $(\mathrm{MH}), \mathrm{Cc}=9.0-$ high degree of cytotoxicity (MT).

We performed four-fold immunization of animals (with an interval of 72 hours, with a volume of $0.1 \mathrm{ml}$ ) subcutaneously in the peritoneal area by serum of patients with different clinical phenotypes of myasthenia gravis. There were 4 groups of animals ( $n=5$ in each group): group I (control) animals which were injected with saline in a volume of $0.1 \mathrm{ml}$; group II (M) - 
animals which were injected by serum with a low degree of cytotoxicity; group III (MH) - animals which were injected by serum with average cytotoxicity; group IV (MT) - animals which were injected by serum with a high degree of cytotoxicity. During the experiment, the physiological indicators of animals were assessed - temperature and weight change. Animals were removed from the experiment by decapitation 20 days after the first immunization.

Determination of the $\mathrm{C} 3$ and $\mathrm{C} 4$ of complement components concentration was performed by immunoturbidimetry (Dialab, Austria) at a wavelength of $340 \mathrm{~nm}$.

All data are presented as the means (x) and standard error (SE). Significant differences between groups were determined by a nonparametric Mann Whitney U test. All statistical analyses were performed using Statistica 6.0 (Statsoft, USA) software pack. Differences between control and experimental groups were considered significant at $\mathrm{P}<0.05$.

Results and discussion. Antigenic exposure is associated with the ability of the antigen to cause certain changes in the body and affect the immune system through appropriate receptors [3, p. 7211]. The reactivity threshold in experimental animals can determine the corresponding changes in innate and adaptive humoral factors of immunoresistance in response to various antigens. We observed a temperature change in rats - as a reaction to the antigenic administration after immunization by sera with varying degree of cytotoxicity. The temperature increased from $\Delta=0.5{ }^{\circ} \mathrm{C}$ to $\Delta=0.9{ }^{\circ} \mathrm{C}$ after each immunization compared with the control group $\left(35.6 \pm 0.09^{\circ} \mathrm{C}\right)$.

An important link between the formation of innate immune responses and adaptive immunity are the complement system proteins, which have an opsonizing effect, as well as adaptive humoral immune responses, which are formed due to the formation of specific antibodies [2, p. 613]. In the control group, the concentration of $\mathrm{C} 3$ component averaged $0.61 \pm 0.07 \mathrm{~g} / \mathrm{l}$, and the concentration of C4 component was at the level $0.091 \pm 0.024 \mathrm{~g} / \mathrm{l}$. In groups of animals which was immunized with serum of low (group II - M) and high (group IV - MT) cytotoxicity, the concentration of C3 component was on average 15\% lower than in the control (group I). And in the group of animals which was immunized with serum of average cytotoxicity (group III - MH), the concentration of $\mathrm{C} 3$ component, on the contrary, was $15 \%$ higher than in the control (group I) - against the background of a significant decrease in temperature $\Delta=0.7{ }^{\circ} \mathrm{C}$ after last immunization. In the same group, we observed a minimal increase in body weight $(\Delta=15 \mathrm{~g})$.

The C4 component complement concentration significantly differed from the control in animals sensitized of serum with low cytotoxicity 
(group II - M), and was 2.3 times higher, which was accompanied by an increase in temperature of animals in this group by the end of the experiment.

Conclusion. Thus, the administration of the antigens complex to the experimental animals led to an immunologically mediated increase in sensitivity - a sensitization state, which reflected the reactivity degree of the organism and the immunogenicity degree of the injected material. It is known that the reactivity of the organism depends on genetic and epigenetic factors that affect the overall functioning of body systems [4, p. 289]. And the initiation of the immunogenic response depends on many factors and signals that increase the efficiency of processing and presentation of the antigen [5, $\mathrm{p}$. 787]. We revealed an increase in the concentration of $\mathrm{C} 3$ (convertase) in the group of animals which were immunized of serum with average cytotoxicity ( $\mathrm{MH}$ - myasthenia with hyperplasia) and $\mathrm{C} 4$ complement component in the group of animals immunized with serum with low cytotoxicity (M myasthenia without thymus changes). This may be one of the manifestations of the inflammatory process, which is characterized by the loss of protective and adaptive function and its transformation into a separate pathogenic factor.

\section{References:}

1. Mahanty S., Prigent A., Garraud O. Immunogenicity of infectious pathogens and vaccine antigens. BMC Immunology. 2015. Vol. 16. No 31. P. 1-6.

2. Foley J. H., Peterson E. A., Lei V., Wan L. W., Krisinger M. J, Conway E. M. Interplay between fibrinolysis and complement: plasmin cleavage of C3b modulates immune responses. Journal of Thrombosis and Haemostasis. 2015. Vol. 13. No 4. P. 610-618.

3. Chen L., Deng H., Cui H., Fang J., Zuo Z., Deng J., Li Y., Wang X., Zhao L. Inflammatory responses and inflammation-associated diseases in organs. Oncotarget. 2018. Vol. 9. No 6. P. 7204-7218.

4. Klimova E. M., Bozhkov A.I., Kovalenko T. I., Minukhin V. V., Belozerov I. V. Young and old animals use different strategies for forming an immune response to infectious agents (Preudomonas aeruginosa and Escherichia coli). Advances in Gerontology. 2018. Vol. 8. No 4. P. 284-291.

5. Ricklin, D., Hajishengallis, G., Yang, K., Lambris, J. D. Complement: a key system for immune surveillance and homeostasis. Nature Immunology. 2010. No 11. P. 785-797. 